\title{
Lyrik und Autobiographik
}

\author{
Zur Funktion von Orts- und Zeitangaben in den Peritexten \\ von Gedichten
}

\section{Lyrische Selbstinszenierung als Herausforderung der Lyrikologie}

Autobiographisch inszenierte Selbst-Aussagen, so genanntes "self-staging", "self-fashioning" (Greenblatt 1980) oder (teils selbst wieder ironisiertes oder konventionalisiertes) `Selbstmaskieren in der eigenen Rolle $\triangleleft,{ }^{1}$ gehören mit zum Repertoire derjenigen Textgruppenbildung, die Leser heute typischerweise als Lyrik zu bezeichnen pflegen. Seit der Antike gibt es eine reiche Lyriktradition, in der sich Dichter gleichzeitig als textexterne Urheber ihrer Gedichte sowie als Referenz des `Ichs in ihren Gedichten inszenieren. Berühmt ist etwa die Doppelrolle von `poeta und `amator`, die das `Ich in Ovids Amores für sich beansprucht (vgl. Fischer 2007, 18-24). Auch im Mittelalter, etwa in der editorischen Verbindung von Troubadourlyrik und Troubadourviten (den so genannten srazos`, vgl. Boutière und Schutz 1964), wird die leserseitige Faszination für die Biographie des empirischen Dichters gezielt angeregt und bedient. Auf diese Tradition der provenzalischen >razos` greift dann etwa Dante in Vita Nova (um 1293) zurück, indem er seine Sonette und Canzonen in die Prosa-Lebenserzählung eines »Ichs « einbettet, das sich selbst Eigenschaften zuschreibt, die auch der reale Autor Dante nachprüfbar und für das zeitgenössische florentinische Publikum bekanntermaßen hatte. Das »Ich« in der Rahmenerzählung von Vita Nova hat also die Wirkungsdisposition, von Rezipienten als Referent für Dante selbst aufgefasst zu werden, und die Aussagen dieses »Ichs« könnten somit prinzipiell als Äußerungen mit Anspruch auf Referenzialisierbarkeit dem realen Dichter zugeschrieben werden. Zudem teilt dieser Rahmen-Icherzähler in Vita Nova für jedes Gedicht, das er in seinen Prosatext einbettet, minutiös mit, bei welchem Anlass und unter welchen

1 Etwa so, wie in modernen Filmen ein Schauspieler sas himself $\triangleleft$ auftreten kann. Dabei bewegt sich der reale Schauspieler nicht metaleptisch in die fiktive, vom Film dargestellte Welt hinein, sondern er spielt im Film nach einem festgelegten Skript sich selbst. Die Rolle könnte also nicht ohne Sinnverlust mit jemand anderem besetzt werden (vgl. dazu etwa Genette 2004, 71, Molières Komödien, oder auch für die Lyrik im Folgenden das Ronsard-Beispiel). 
Umständen, ja sogar für welches Publikum und in welcher Absicht er seine Verse jeweils verfasst habe. So lautet zum Beispiel die Einleitung im Falle des ersten Gedichtes der Sammlung, nachdem der Erzähler von einer nächtlichen Vision berichtet hat:

Und dieweil ich über diese Erscheinung nachsann, nahm ich mir vor, sie vielen kund zu tun, die zu jener Zeit geachtete Sänger und Dichter waren. Und [...] [so] beschloss ich, ein Sonett zu dichten, worin ich mich an alle Liebestreuen wenden wollte, und indem ich sie bat, mir mein Gesicht zu deuten, beschrieb ich ihnen, was ich gesehen hatte, und begann dies Sonett: [...] (Dante 1947, 13 und 15) ${ }^{2}$

Ob wiederum der diese Worte äußernde Rahmen-Icherzähler in Dantes Text eine fiktive Erzählerfigur ist oder nicht, sei hier einmal dahingestellt (vgl. dazu Näheres z. B. bei Kablitz 2009). In jedem Fall behauptet dieser Erzähler aber, dass er die Gedichte verfasst habe (er also der praktische Ausgangspunkt des lyrischen Sprachzeichengebildes sei) und dass das >Ich heißt, dass die Gedichte - bei aller Künstlichkeit der sprachlichen ıÜberstrukturierung`, das heißt der genretypisch komplexen Organisation von `Faktur ‘ und ıInformation` (vgl. Zymner 2017, Glossar, 4) - autobiographische Texte seien.

Bei Dante wird dieser Anspruch auf Autorfaktualität der Lyrik (vgl. Zymner 2009, 12) in der die Gedichte rahmenden (und vielleicht selbst fiktionalen) Prosaerzählung erhoben. Eine zweite Möglichkeit der autobiographischen Inszenierung von Lyrik, das heißt der Anbietung einer Referenz von gedichtinternem >Ich ‘ auf realen Textproduzenten, läuft über die Verwendung nicht-fiktionaler Elemente wie zum Beispiel Personennamen im lyrischen Schriftzeichengebilde selbst. ${ }^{3}$ So verwendet etwa Ronsard seinen eigenen Namen sowie in poetisierter Form auch

2 »Pensando io a ciò che m'era apparuto, prospuosi di farlo sentire a molti li quali erano famosi trovatori in quello tempo: e con ciò fosse cosa che io avesse già veduto per me medesimo l'arte del dire parole per rima, propuosi di fare uno sonetto, ne lo quale io salutassi tutti li fedeli d'Amore; e pregandoli che giudicassero la mia visione, scrissi a loro ciò che io aveva nel mio sonno veduto. $\mathrm{E}$ cominciai allora questo sonetto, lo quale comincia: [...].« (Dante 1947, 12 und 14.)

3 Vgl. zu Personennamen den Beitrag von Dieter Burdorf zum vorliegenden Band. Allerdings berücksichtigt Burdorf (zum Beispiel im Celan-Beispiel »Lies, Ingeborg, lies «) auch sallographe (im Falle der Celan-Widmung also >private und erst postum im Nachlass auffindbare) Epitexte. Ob man solche Epitexte >jenseits ‘ des Artefakts für die Interpretation als berechtigterweise heranzuziehen einstuft oder nicht, hängt von der interpretationstheoretischen Vorannahme ab. Ich beschränke mich hier auf Gedichte als Artefakte in ihren Publikationsmedien und berücksichtige nur die mit den Gedichten zusammen veröffentlichten Peritexte, um mein Theoriemodell zunächst interpretationstheoretisch möglichst unabhängig (etwa von der Frage nach der Berücksichtigung von saktualen Autorintentionen`) zu halten. 
den der mutmaßlichen Widmungsadressatin Françoise Babou d'Estrées (vgl. Ronsard 2010, 264) in seinen »Sonnets et madrigals pour Astrée« (1578):

\author{
[...] \\ Et moi je veux honorer ma contrée \\ De mon sépulcre et dessus engraver : \\ Ronsard, voulant aux Astres s'élever, \\ Fut foudroyé par une belle Astrée. (Ronsard 2010 [1578], 216; Hervorhebungen S. K.)
}

Eine dritte Möglichkeit, eine Referenz von gedichtinternem `Ich` auf den realen Autor anzubieten, besteht in der Kombination des Gedichttextes mit konkreten Orts- und Datumsangaben in den Peritexten, die - wie etwa im Falle von Goethes Gedicht mit dem Titel »Dornburg, Septbr. 1828« - nachprüfbar Stationen auf dem Lebensweg des empirischen Autors benennen. In der Interpretationsgeschichte von Goethes Gedicht ist diese autobiographische Verbindung von Orts- und Zeitangabe meist gedeutet worden als Referenz auf den Aufenthalt des fast schon greisen Dichters in der herbstlichen Natur der Saale-Landschaft.

Inszenierungen eines autobiographischen Referierens von Lyrik gehören also unstrittig durchaus zum Kanon des Genres Lyrik, auch wenn die hier illustrierte Dreierliste, auf welche Arten eine Referenz des >Ichs ‘ im Gedicht auf den realen Autor angeboten werden kann, vermutlich noch nicht vollständig ist. Unbestritten ist in der Forschung weiterhin, dass es Gedichtarten wie etwa das Rollengedicht oder die "poetische Grabschrift « ${ }^{4}$ gibt, in denen das gedichtinterne >Ich eindeutig nicht auf den empirischen Autor referiert. ${ }^{5}$ Unbestritten ist ebenfalls, dass es Gedichte gibt, in denen durch die Verwendung bestimmter sprachlicher Zeichen ein "pragmatischer Ausgangspunkt[...] des lyrischen Sprachzeichengebildes « markiert wird. Zu diesen sprachlichen Markierungen gehören vor allem Personalpronomina in der ersten Person, entsprechend flektierte verba dicendi, agendi oder auch sentiendi sowie deiktische Ausdrücke, die auf eine Ich-HierJetzt-Origo bezogen sind. Für den solcherart markierten "pragmatische[n] Ausgangspunkt[...] des lyrischen Sprachzeichengebildes« (also des Gedichtes) im

\footnotetext{
4 Hier wird typischerweise die mentale Repräsentation der offenkundig fiktiven Sprechsituation getriggert, in der der Tote aus dem Grab heraus einen vorübergehenden Wanderer anspricht (vgl. Segebrecht 1978, Braungart 1997, Klimek 2013).

5 Je nachdem, ob die Aussagen in solchen Gedichten Anspruch auf Erfülltheit in der Wirklichkeit erheben oder nicht, spricht Zymner (2009, 12-15) von "persona-faktualen« oder »persona-fiktionalen« Gedichten. - Zum Verhältnis von Lyrik und Figur, vgl. den Aufsatz von Claudia Hillebrandt zum vorliegenden Band.
} 
Gedicht selbst verwende ich den im Glossar zum vorliegenden Band von Zymner vorgeschlagenen lyrikologischen Begriff `Adressant`.

Im Folgenden geht es vor allem um den von Zymner identifizierten Sonderfall, dass zwar ein Adressant im Gedicht markiert wird, aber aufgrund des Textbefundes nicht entscheidbar ist, ob dieser Adressant die Vorstellung einer fiktiven oder realen Äußerungssituation triggert (in Zymners Terminologie: ‘adressantenneutrak).

Dieser Sonderfall ist insofern interessant, als in der gegenwärtigen Lyriktheorie umstritten ist, ob das in einem Gedicht vorkommende sprachliche Zeichen >Ich überhaupt auf den empirischen Autor referieren kann (das heißt auf die reale Person, die das Gedicht »formuliert und formatiert«, Zymner 2017, Glossar), und ob lyrische Sprachzeichen überhaupt als Repräsentationen von Aussagen aufgefasst werden können, die einen Anspruch auf Referenzialisierbarkeit oder Erfülltheit in der Wirklichkeit erheben (sautor-fiktionale` oder auch sautor-faktuale` Gedichte, Zymner 2009, 12-15).

Zusätzlich zu diesen gegenwärtig zu beobachtenden, entgegengesetzten interpretationstheoretischen Vorannahmen zum Verhältnis von Fiktionalität und Lyrik ist jedoch auch in der Lyrik selbst eine Variabilität der historischen Konventionen, Anspruch auf Referenzialisierbarkeit zu codieren, bemerkbar. Daher plädiert Lampart dafür, die Dichotomie von Fiktionalität und Faktualität in der Lyriktheorie zugunsten von "Differenzierung und offene[n] Skalen « aufzugeben, stattdessen eine "historisch abgewogene Skalierung von autorfaktualen Elementen und Anteilen einer rhetorisch-stilistischen Gestaltung zu rekonstruieren « und im Einzelfall »zumindest als Untersuchungshypothese die Möglichkeit eines Nebeneinanders autorfaktualer Referenzialisierbarkeit und referenzfreier, sfiktionaler Textschichten in Betracht« zu ziehen. ${ }^{6}$ Dieses Plädoyer scheint mir auch für die Erforschung von Gedichten mit autobiographisch inszenierenden Peritexten sinnvoll zu sein.

In der Literaturwissenschaft gibt es unterschiedliche interpretationstheoretische Vorannahmen darüber, inwiefern ein Eingehen auf die Biographie des Verfassers berechtigt sei. ${ }^{7}$ Die literaturwissenschaftliche Autobiographie-Forschung hat sich, unter dem Einfluss von Lejeunes $(1994,14)$ wirkmächtiger Definition der Autobiographie als der »rückblickenden Prosa-Erzählung einer tatsächlichen Person über ihre eigene Existenz« (meine Hervorhebung, S. K.), bisher nicht pro-

6 Vgl. Fabian Lamparts Beitrag zum vorliegenden Band.

7 Zur Unterscheidung von `biographischer Methode` und unangemessenen `biographistischen Lesarten, vgl. grundsätzlich Kindt und Müller (2002). 
minent der Lyrik mit autobiographisch inszenierenden Peritexten angenommen. Es existiert bis anhin kein Beschreibungsmodell, das - möglichst unabhängig von interpretations-, aber vor allem auch von fiktionstheoretischen Positionen des zwanzigsten und einundzwanzigsten Jahrhunderts - dazu fähig wäre, autobiographische Peritexte von Gedichten (und nur auf diesen oben beschriebenen dritten Fall werde ich im Folgenden eingehen) methodisch abgesichert in die Analyse einzubeziehen, ohne dabei dem grundsätzlichen und berechtigten Misstrauen gegen ein unreflektiertes Gleichsetzen von empirischem Autor und gedichtinternem »Ich« Anlass zu geben. Im Folgenden soll die Notwendigkeit eines solchen Theorie-Modells anhand von vier Gedichtbeispielen aus dem siebzehnten, achtzehnten und frühen neunzehnten Jahrhundert illustriert und ein entsprechendes Modell skizziert werden.

\section{Wer spricht das Gedicht? Bisherige Modelle, Theorien, Begriffe und Konzepte}

„Wer spricht das Gedicht?« (Borkowski und Winko 2011) - Häufig wird diese Grundfrage der Lyrikologie in einer metaphorischen Redeweise als Frage nach der ১Stimme` (im Sinne Genettes 1994 [1972]), bzw. nach der gedichtinternen Äußerungsinstanz, eventuell sogar nach der typisch oder gar prototypisch lyrischen Konstitution der mentalen Repräsentation einer textinternen Kommunikationsmatrix gestellt. Jedoch muss die Frage an sich bereits als problematisch gelten, ${ }^{8}$ ist doch Lyrik in vielen ihrer heute verbreiteten Erscheinungsweisen - mit Ausnahme beispielsweise der so genannten `Oral Poetry - eben nicht in Form von gesprochener Sprache repräsentiert, bei der eine sprechende Stimme zu hören wäre, sondern meist (aber nicht notwendigerweise) in Form von gedruckter Sprache, die häufig nur von der sinneren Stimme` des betreffenden Rezipienten ' gesprochen ‘ wird. ${ }^{9}$ In der Narratologie ist diese Problematik der metaphorischen Rede von akustischen Instanzen wie der `Stimme auf rein visuelle, das heißt nur durch Schrift konstituierte Artefakte bereits eingehend reflektiert worden (vgl. dazu etwa Blödorn, Langer und Scheffel 2006). Erzähltexte stellen - so die weithin akzeptierte Ansicht - nur eine Art Simulation des >natürlichen`, alltagssprachlichen, mündlichen Erzählens im Medium der Schrift dar. Wie aber verhält es sich mit Gedichten? Simuliert die Lyrik auch

8 Vgl. die Einleitung zum vorliegenden Band.

9 Vgl. dazu auch Rüdiger Zymners Beitrag über Animismus im vorliegenden Band. 
etwas? Falls ja, was? Alltägliches Sprechen scheint es aufgrund ihrer speziellen Faktur, ihrer oft hochartifiziellen Rhetorik und der besonderen Bedeutung, die klanglichen Aspekten ja auch in nur schriftlich fixierter Lyrik zukommt, gerade nicht zu sein. Und auch das Erzählen ist trotz einiger prominenter Vorschläge, die Lyrik als seine >Devianzform ‘ zu definieren (vgl. etwa Hühn und Schönert 2007, 4), wohl nicht transhistorisch als gattungskonstitutives Merkmal stark zu machen. Klar ist darüber hinaus, dass sich die Frage nach einer vom Gedicht getriggerten Vorstellung einer >Sprech/-Instanz für visuelle Poesie erübrigt. Wo jedoch innerhalb der Lyrik Textlichkeit vorliegt, ${ }^{10}$ ist es die Wirkungsdisposition des Sprachzeichengebildes Gedicht, dass seine Rezeption beim Rezipienten die mentale Vorstellung (das heißt ein `kognitives Konzept` und keinen >Adressanten`, denn der ist ja in Zymners Begriffssystem nur eine Zeichenstruktur im Text) einer anthropomorphen >Äußerungsinstanz` hervorruft. Dieser kann der Rezipient zuschreiben, Ursprung der sprachlichen Äußerungen zu sein, als die er die Sprachzeichen des Gedichts auffassen kann. ${ }^{11}$

Die Frage »Wer spricht das Gedicht?« ist zudem nicht unabhängig davon zu beantworten, welchen Umfang man dem zugrunde liegenden >Gedichtı-Begriff beimisst. Zum Begriff der Lyrik gibt es verschiedene, konkurrierende Definitions- und Explikationsvorschläge - wenn man denn überhaupt die heute alltagssprachlich oft synonym verwendeten Begriffe >Gedichte und 〉Lyrik« (mit Burdorf 2015, 20) als deckungsgleich bezeichnen möchte. Legt man beispielsweise einen etwas weiteren Lyrik-Begriff an, für den Literarizität kein notwendiges Kriterium ist, es Lyrik also auch »vor, neben und außerhalb« dessen gibt, was in Europa

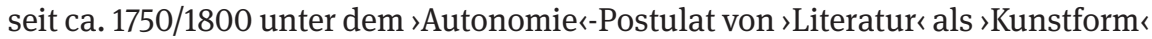
produziert und verstanden wird (vgl. Zymner 2013, 27), so muss die Lyrikologie fähig sein, auch »situationsbezogene« (Segebrecht 1978) Gedichte umfassend zu beschreiben. Daraus folgt, dass man mit Konzepten, die 1:1 aus der Theorie fiktionaler Dramen- oder Erzähltexte übernommen sind, sautor- $\iota$ wie spersona-faktuale ${ }^{12}$ (oder zumindest als faktual inszenierte) Gedichte nicht adäquat erfassen

10 Dass es auch Lyrik ohne Textlichkeit, das heißt »Redezeichengebilde unterhalb der Textebene« wie Laut- oder Einwortgedichte geben kann (vgl. Zymner 2009, 32), sei damit nicht bestritten.

11 Zur Frage, ob tatsächlich jedes fiktionale (auch impersonal-heterodiegetische) Erzählen die Annahme einer fiktiven Erzähler-Figur bedinge oder ob es auch `erzählerloses Erzählen` geben könne, vgl. Köppe und Stühring (2011).

12 Zymner (2009, 11-12); vgl. auch Lampings (2000 [1989], 108) Unterscheidung von »pragmatischer« und »semantischer Fiktionalität«. 
kann. ${ }^{13}$ Gerade dies müsste eine interpretationstheoretisch möglichst neutrale Lyriktheorie aber leisten können. Man muss nicht gleich mit Hamburger (1968) und Culler (2015) verallgemeinern, dass Lyrik - zumindest dort, wo keine Rollenfiktion explizit markiert ist - trotz aller >Ritualität ` des sprachlichen Ausdrucks »Wirklichkeitsaussage« sein und etwas Wichtiges »about meaning and value« $\mathrm{zu}$ sagen habe. Lyrik scheint aber, wenn auch nicht konstitutiv, so doch zumindest in gewissen historischen Kontexten und/oder Genres, die Wirkungsdisposition zu haben, dass Rezipienten davon ausgehen, dass in ihr (ob nun tatsächlich behauptend oder nur rollenhaft/fiktional) >der Dichter als er selbst spricht‘, weil im Gedicht oder seinen Peritexten eine Referenz des Sprachzeichens >Ich Gedicht auf den realen Autor des Gedichtes angeboten wird.

Oft wird in Bezug auf Lyrik der hohe Grad sprachlicher Stilisierung als Argument dafür genannt, dass Lyrik immer fiktional sein müsse. So etwa von Fischer (2007, 11), die sich fragt, warum eigentlich gewisse Merkmale von Gedichten »immer wieder und bis heute dazu führen, dass die Aussagen eines Ich in einem lyrischen und damit in höchstem Maße artifiziellen Text immer wieder auch auf dessen Autor bezogen werden [...], [was] die Trennung zwischen Text- und Autorenebene immer wieder verschwimmen lässt [...].« Hinter Fischers unausgesprochener Vorannahme, das `Ich` in einem Gedicht könne niemals auf den empirischen Autor verweisen, steht offenbar die ebenfalls unausgesprochene Vorannahme, in einem »in höchstem Maße artifiziellen Text« könne grundsätzlich nicht auf die textexterne Wirklichkeit verwiesen werden. Nicht-Fiktionalität schließt das kunstvolle Stilisieren jedoch ebenso wenig aus wie das Übertreiben und das rollenhafte Inszenieren, ja nicht einmal das Lügen, denn all diese Redeweisen erheben - im Unterschied zur fiktionalen Rede - Anspruch auf Referenzialisierbarkeit, das heißt auf Erfülltheit in der textexternen Wirklichkeit (sind also nicht-fiktional), ohne dass damit tatsächlich durchweg wahre Aussage getätigt sein müssten (vgl. Gabriel 1997, 595). Auch politische Reden beispielsweise können rhetorisch »in höchstem Maße artifizielle Text[e]« sein und erheben dennoch Anspruch auf Referenzialisierbarkeit ihrer Aussagen. Und auch appellative Sprechakte kann man durchaus in Form eines kunstvollen Gedichtes tätigen. Wenn man beispielsweise die Sätze »Deshalb liebe ich dich. / Bitte heirate mich!« am Ende eines Liebesgedichts als fiktionale Äußerungen einer fiktiven Figur innerhalb der >Storyworldı eines autonomen Kunstwerks interpretiert und entsprechend der Fiktionalitätshypothese nur auf das anapästische Metrum und den nicht sehr kreativen Endreim dieses Zweizeilers eingeht, kann dieses Ver-

13 Vgl. zu diesem Problem neben Burdorfs Aufsatz auch die Beiträge von Trilcke und Lampart zum vorliegenden Band. 
halten - je nach Vortragssituation - durchaus eine unangemessene, jedenfalls nicht die vom Sprecher erhoffte Reaktion sein.

Wie schon die Frage »Wer spricht?« eine Übertragung von Genettes erzähltheoretischer Kategorie der >Stimme ‘ in die Lyrikanalyse andeutet, so wurden generell in den letzten zwei Jahrzehnten verschiedene Modelle, Theorien, Begriffe und Konzepte aus der >klassischen ` Narratologie in die Lyriktheorie importiert, um Gedichte künftig durch eine exaktere Beschreibungssprache präziser analysierbar (und gegebenenfalls somit besser interpretierbar oder intensiver genießbar) zu machen. Einige Arbeiten zielten etwa darauf ab, die Informationsvergabe bzw. Perspektivierung des im Gedicht Dargestellten analog zur Fokalisierung in Erzähltexten klassifizierbar zu machen (vgl. Petzold 2012). Andere setzten dazu an, die Staffelung der verschiedenen >Aussageinstanzen` (empirischer Autor, impliziter Autor, fiktiver Sprecher/Erzähler und fiktive Figuren) auf die Lyrik zu übertragen (vgl. Wolf 1998, Schönert 2004, Müller-Zettelmann 2002, im Prinzip auch Burdorf 2015, 194-201). ${ }^{14}$ Doch es gibt auch genuin aus der Lyrikanalyse und -interpretation hervorgegangene Modelle, in denen eine vom Gedicht angestoßene Vorstellung einer Kommunikationsmatrix unter Rückgriff auf linguistische Modelle der Pragmatik beschrieben wird, wie Semino (1997), Schiedermair (2004), Borkowski und Winko (2011, 64-75). Gerade im englischsprachigen Raum war die Vorstellung von >lyric als einer immer fiktionalen Äußerungssituation mit einem fiktiven Sprecher als ıspeaker des Gedichttextes (vgl. etwa Wolf 1998, Müller-Zettelmann 2002) lange Zeit vorherrschend. Sie wird erst in jüngerer Zeit wieder hinterfragt, etwa von Culler $(2015,2)$. Dieser argumentiert, dass Ansätze, welche Lyrik über die (narratologisch oder dramentheoretisch orientierte) Fiktionstheorie erschließen, die auffälligsten Merkmale von lyrischen Texten überspielen (»the most salient features of many lyrics«; Culler 2015, 350). Culler (2015, 350) folgt Hamburger $(1968,188)$, wenn er betont, dass Lyrik im Grunde genommen eine Aussage über die Welt sei (»lyric is, at bottom, a statement about this world rather than the projection of a fictional speaker and a fictional world «). Lyrik lässt sich für ihn als ein öffentliches Sprechen über Bedeutung und Werte mithilfe von ritualistischen Elementen verstehen (»public discourse about meaning and value - made distinctive by its ritualistic elements«; Culler 2015, 350). Ins Zentrum der Gattung Lyrik stellt Culler (2015, 15-16) somit jene Gedichte, die sich durch eine »triangu-

14 Vgl. dazu den Aufsatz von Evelyn Dueck im vorliegenden Band. Für eine kritische Sichtung, vgl. Hillebrandt 2015. Für die Kritik, eine von der Erzähltextanalyse ausgehende Modellierung lyrischer Sprechinstanzen übersehe gerade die generisch andere, nämlich "prototypisch lyrische Äußerungsstruktur«, vgl. Hempfer $(2014,17)$. 
lated address« auszeichnen: Viele Gedichte richten sich nämlich, so Culler, an ihre tatsächlichen Empfänger, indem sie als >Adressaten ` eine abwesende Instanz (zum Beispiel Gott, einen Geliebten, Unbelebtes wie die Sonne, den Mond, Berge, oder Abstrakta, wie den Tod, etc.) ansprechen, die in der Regel im Gedicht nicht antwortet. Aber macht eine solche striangulierte، Anrede ein lyrisches Sprachzeichengebilde bereits zu einem fiktionalen Text?

Die Frage nach der möglichen oder gar generisch unhintergehbaren Fiktionalität von Lyrik wurde gerade im deutschsprachigen Raum heftig diskutiert (für einen neueren Überblick, vgl. Zipfel 2011). Typisch für die deutsche Debatte ist dabei der Streit um den bereits 1910 von Margarete Susman in die Literaturwissenschaft eingeführten Begriff des >lyrischen Ichs`. Susman $(1910,19)$ sprach offen aus, dass ihr Modell lyrischen Sprechens auf einem Analogieschluss von fiktiven Figuren im Drama auf die durch Lyrik getriggerte Vorstellung einer Äußerungsinstanz im Gedicht beruhe: »Der Dichter findet dieses Ich nicht in sich vor, sondern ähnlich den redenden und handelnden Gestalten eines Dramas muß er auch das lyrische Ich erst aus dem gegebenen erschaffen."

Der Begriff des >lyrischen Ichs` wird in der literaturwissenschaftlichen Praxis z. T. bis heute in diesem Sinne benutzt, um den postuliert fiktiven (das heißt in Susmans Worten »erschaffenen«), im Gedicht markierten Adressanten vom realen Autor als dem empirischen Textproduzenten abzugrenzen. Müller (bereits 1979; erneut 2011, 57) ${ }^{15}$ dagegen argumentiert etwas vorsichtiger, wenn er betont, lyrisches Ich und empirischer Autor seien zwar in der Analysepraxis zunächst einmal »strikt zu trennen«, doch könne damit »nicht ausgeschlossen sein, dass das lyrische Ich vielfach auf eigenen Erfahrungen des Dichters aufbaut«. Der Begriff 〉lyrisches Ich ist jedoch mit wechselnden Begründungen auch abgelehnt worden, etwa von Burdorf $(2015,192)$, der wegen der »[v]erworren[en]« Begriffsgeschichte das `lyrische Ich` heute für problematisch hält und es lieber durch den unvorbelasteten neuen Terminus »artikuliertes Ich« (Burdorf 2015, 194) ersetzen möchte. ${ }^{16}$ Fricke und Stocker (2000) versuchen dagegen, den Begriff des »Lyri-

15 Bei Müller $(2011,58)$ finden sich auch einige der seltenen Hinweise zur Rezeption und Diskussion des Begriffs außerhalb der Germanistik, etwa bei Rabaté 1996 (»sujet lyrique«) und Blasing 2006. Vgl. dazu auch Rabaté, de Sermet und Vadé (1996).

16 Burdorfs elaboriertes Modell von Kommunikationssituationen der Lyrik unterscheidet zwischen der Rede fiktiver Sprecher in der dritten Person sowie der Rede fiktiver Figuren in der ersten Person, zwischen einem im Gedicht sich ohne vermittelnde Erzählerstimme direkt äußernden 'Rollen-Ich und einem durch nichts als Rolle markierten, unvermittelt sprechenden Ich, das er als sartikuliertes Ich ‘ bezeichnet. Diese letzte Kategorie Burdorfs scheint mir vom Begriffsumfang her deckungsgleich zu sein mit der von Fricke und Stocker (2000) vorgeschlagenen Explikation des Begriffs `Lyrisches Ich` als Sprecher-Ich in Gedichten ohne erkennbare »Rollenfiktion«. 
schen Ichs « zu rehabilitieren, indem sie auf Grund einer detaillierten Rekonstruktion von Begriffs-, Sach- und Forschungsgeschichte eine präzise Explikation des Begriffs als »Subjekt von Gedichten ohne Rollenfiktion« für den künftigen literaturwissenschaftlichen Gebrauch vorschlagen. Sie grenzen das Konzept explizit ab von epischen und dramatischen Theoriemodellen, indem sie in Hinsicht auf den logischen Status der lyrischen Rede eines solchen sSubjekts ‘ betonen, dass die >Fiktionalität` von Gedichten mit einer Äußerungsinstanz ohne erkennbare Rollenfiktion - im Sinne Hamburgers - unbestimmbar sei, das heißt "gleichsam in der Schwebe« bleibe (Fricke und Stocker 2000, 509). ${ }^{17}$

Das letztlich etwas Unbefriedigende an diesen durchaus hilfreichen Modellen und Vorschlägen ist erstens, dass sie - mit Ausnahme dessen von Borkowski und Winko, welche darüber hinaus auch reale Vortragssituationen in den Blick nehmen und insofern auch die wechselnden sempirischen Sprecher eines Gedichtes mitberücksichtigen ${ }^{18}$ - lediglich die Frage nach der vom Gedicht angestoßenen Vorstellung einer Äußerungsinstanz behandeln (welche von den Rezipienten abhängt), ohne zunächst erst einmal das Gedicht als lyrisches Schriftzeichengebilde als solches - vorgängig aller von ihnen getriggerten Vorstellungen - beschreibbar zu machen. Zweitens berücksichtigen alle bisher referierten Modelle lediglich den Haupt- (das heißt fürs siebzehnte und frühe achtzehnte Jahrhundert meistens: Vers-)Text eines Gedichts. ${ }^{19}$ Gedichte sind und waren jedoch mitnichten derart autonome Gebilde, dass sie nur in Form ihres sunbekleideten Verskörpers` begegnen würden. Wenn jemand sich beispielsweise anschickt, ein Gedicht zu rezitieren, so sagt er meist zunächst den Namen des Verfassers und den Titel des Gedichtes, ehe er den seigentlichen` Gedichttext intoniert. ${ }^{20}$ Und selbst in aktuellen wie historischen Lesebüchern, Almanachen,

17 Für einen noch recht aktuellen Überblick über die Debatte im deutschsprachigen Raum, vgl. Borkowski und Winko (2011, 45-52).

18 Zur Frage nach den Sprechinstanzen im Gedichtvortrag, den ich hier komplett ausklammere, vgl. den Beitrag von Frieder von Ammon im vorliegenden Band.

19 Für die von mir hier behandelten Gedichte kommt man tatsächlich mit Lampings relativ engem Begriffsverständnis des »lyrischen Gedichtes« als »Einzelrede in Versen« (Lamping 2000 [1989], 23) aus.

20 Analog werden auch Popsongs im Radio oder auf TV-Musiksendern meist mit der Nennung des ausführenden Künstlers bzw. der Gruppe und des Titels angekündigt. Der Name des Songwriters tritt dagegen hinter den des Performenden zurück, was meines Erachtens dafür spricht, dass Popsongs anders `benutzt` werden als Gedichte. Für das Kunstlied gelten dagegen wieder andere Konventionen: Auf Klassiksendern nennt die Anmoderation meist den Titel des Stücks und Namen des Komponisten sowie der interpretierenden Orchester oder Solisten. 
Anthologien und Zeitschriften erscheint das graphisch repräsentierte lyrische Sprachzeichengebilde stets in materieller Verbundenheit mit anderen Texten, die zum Teil die Wirkungsdisposition haben, als auf den Gedichttext bezogen verstanden zu werden. Gerade in solchen Neben- oder Peritexten von Lyrik finden sich aber nicht selten die Inszenierungen einer Referenz von >Ich « im Gedichttext auf den realen Textproduzenten bzw. eine Station seines Lebensweges, das heißt das Angebot eines spacte autobiographique (Lejeune) auch für die Lyrik. Die Konventionen, diesen Pakt anzubieten, können sich historisch wandeln (und müssen daher für eine historisch-hermeneutische Interpretation zunächst einmal rekonstruiert werden, wie Fabian Lampart vorschlägt). Ein realer Autor bzw. seine Herausgeber und sonstige am Druckprozess beteiligte Personen können diese Peritexte ein und desselben lyrischen Sprachzeichengebildes jedoch auch von >Textereignis` zu `Textereignis`(Zymner 1995, 40) verändern. Daher möchte ich Fabian Lamparts Plädoyer hinzufügen, dass für eine solche Gedichtinterpretation zusätzlich auch die Textgenese bzw. die Druckgeschichte berücksichtigt werden sollten, denn verschiedene Textereignisse eines einzigen Textes können durchaus voneinander abweichende Wirkungsdispositionen gehabt haben.

\section{Orts- und Zeitangaben als nicht-fiktionale Elemente in den Peritexten von Gedichten}

Bei Gedichten, die vorrangig nicht im mündlichen Vortrag, sondern als gedruckte Texte rezipiert werden, kommen neben Titel- und Verfasserangabe noch weitere Peritexte, manchmal auch Bilder, ja sogar bedeutungstragende druckgraphische Elemente wie die besondere Gestaltung der Schriftbildfläche hinzu, die die eigentlichen Texte >rahmen « und insofern auf die Rezeption der Gedichte Einfluss haben (vgl. Genette 2001, 10-12). Zu den klassischen Peritexten der Lyrik gehören Gedichtüber- und -unterschriften, Motti, Abteilungs- oder Zyklustitel in Gedichtsammlungen, Einleitungen, erklärende Fußnoten, Widmungsadressen, Vor- und Nachworte, etc.

Dieter Burdorf macht in seinem Beitrag zum vorliegenden Band darauf aufmerksam, dass trotz des durchaus vorhandenen Angebots elaborierter Modelle ${ }^{21}$

21 Wie zum Beispiel Borkowskis und Winkos, das dezidiert "voraussetzungsarm und umfassend« ist, indem es auf »gattungsnormative Vorgaben [...] ebenso verzichte[t] [...] wie auf Setzungen des Typs >Lyrik ist immer (oder nie) fiktional oder `Das lyrische Ich ist immer vom Autor zu unterscheiden`.« (Borkowski und Winko 2011, 76-77). 
zur Klassifizierung der von Lyrik getriggerten Vorstellungen von Äußerungssituationen bis heute ein Modell fehlt, das auch fähig ist, Namen in den Paratexten von Gedichten zu berücksichtigen. Ähnlich verhält es sich meines Erachtens mit konkreten Orts- und Zeitangaben in den (gedruckten) Peritexten eines Gedichtes, wenn sich diese nachprüfbar auf Stationen des Lebensweges des empirischen Autors beziehen. So etwa im Falle der beiden >Dornburger Gedichte` von Johann Wolfgang von Goethe, »Dem aufgehenden Vollmonde« mit dem Untertitel »Dornburg, Aug. 1828« (Goethe 1833, 66) ${ }^{22}$ und »Dornburg, Septbr. 1828« (Goethe 1833, 68). Bei letzterem ist die Orts- und Zeitangabe sogar die einzige Gedichtüberschrift. Doch ist diese Überschrift in der Ausgabe letzter Hand in derselben, nämlich etwas kleineren Schriftgröße gesetzt wie zwei Seiten vorher der Untertitel von »Dem aufgehenden Vollmonde ${ }^{23}$ Berücksichtigt man dieses druckgraphische Element, so hat das erste `Dornburger Gedicht` (diese Sammelbezeichnung stammt übrigens nicht von Goethe) einen Haupt- und einen Untertitel, das zweite dagegen besitzt nur einen Unter- und keinen Haupttitel. Allerdings ist für den 47. Band der Ausgabe letzter Hand nicht bekannt, ob Goethe hier noch Fahnen gesehen hat, das heißt, ob die Schriftgrößenwahl überhaupt vom Autor autorisiert bzw. ob sie ein verstehensrelevanter Teil der Faktur des Gedichtes ist. Dies kann allein auf Grund des philologisch gesicherten Textbefundes nicht geklärt werden, sondern ist bereits ein (notwendigerweise von interpretationstheoretischen Vorannahmen bestimmtes) Ergebnis einer Interpretation.

22 Je nach Textzeugen mit oder ohne konkrete Tagesangabe des 25., vgl. dazu auch Klimek (2016).

23 Das erste der beiden >Dornburger Gedichte wurde in der Ausgabe letzter Hand zum ersten Mal veröffentlicht, das zweite erschien jedoch auch schon vorab, zusammen mit drei anderen vorher unpublizierten Gedichten Goethes, im Deutsche[n] Musenalmanach für das Jahr 1833 (7). Goethe hat die beiden über die Orts- und Zeitangabe mit seinem Dornburger Aufenthalt verbunden Gedichte also mitnichten als Einheit oder gar als Mini-Zyklus publiziert. Vielmehr rückte er zwischen beide in der Ausgabe letzter Hand das Gedicht »Der Bräutigam« (Goethe 1833, 67) ein. Die Datierung von »Der Bräutigam« ist in der Forschung zwar umstritten (vgl. Eibl 1988, 1221), doch wird gemeinhin nicht davon ausgegangen, dass dieses Gedicht während Goethes DornburgAufenthalt 1828 entstanden ist oder inhaltlich etwas mit den beiden >Dornburger Gedichten $`$ zu tun hat. 


\section{Dem aufgehenden Bollmonde.}

Dornburg, 25 भug. 1828.

FBiflit bu mid fogleid) verlaffen?

Warft im 2ugenblid to nah!

Did) umfinitern $\mathfrak{B o l t e n m a f i e n , ~}$

und nun bift bu gar nidt ba.

Dods bu futblift wie id betribt bin, Bliat bein Rand berauf als Sten! Seugeit mir baß id geliebt bin, Sey bas qiebden now fo fern.

So binan benn! Gell und beller, 'Reiner $\mathfrak{B a h n , ~ i n ~ v o l l e r ~ p r a d t ! ~}$

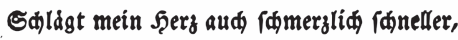

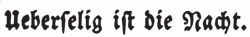

Dornburg, Gept6r. 1828.

Frail wenn Thal, Bebirg unb Barten Rebelí)leiern fí enthüllen, Ind bem Pehnlidiften Erwarten Blumentelde bunt fíd fúlten;

Baenn ber Mether, Bolfen tragenb, Mit Dem Ilaren Iage ftreitet, und ein Dotuvinb, fie veriagend, Blaue Sonnenbaln bereitet;

Danfit bu bann, am Blid bia weibend, Reiner Bruft Der Grofen, F̧olben, Siirb bie Sonne, rottlia faeibenb, æings ben Şorizont vergolden.

Abb. 1 und Abb. 2: Goethe's Werke. Vollständige Ausgabe letzter Hand. Band. 47. Stuttgart und Tübingen: Cotta, 1833. 66 und 68.

Ein kurzer Blick in die reiche Forschungsliteratur zu Gedichten wie Goethes >Dornburgern zeigt, dass solche Texte, die über Orts- und Zeitangaben als ıautobiographisch motiviert ^ oder zumindest `autobiographisch eingebettet ` inszeniert sind, tatsächlich häufig mit Bezug zum Leben des realen Dichters interpretiert werden. Immer wieder erwähnt werden etwa Goethes Trauer um den kurz vor dem genannten Datum verstorbenen Herzog Karl August von Sachsen-WeimarEisenach, Goethes Beziehungen zu Marianne von Willemer und dem Briefpartner Zelter sowie intertextuelle Bezüge zu möglichen Parallelstellen in anderen Texten desselben Autors - zu literarischen wie dem West-östlichen Divan, naturwissenschaftlichen wie der Farbenlehre, ${ }^{24}$ aber auch zu privaten wie den in seinem Tage-

24 Exemplarisch genannt seien hier Schöne (1979) und Detering (2009) sowie der Kommentar von Eibl (1988). 
buch des Dornburg-Aufenthalts vom 7. Juli bis 11. September 1828 vermerkten Wetterbeobachtungen. ${ }^{25}$ Eines der ersten Ziele einer systematischen Lyrikologie sollte es nicht sein, solche Interpretationspraktiken $\mathrm{zu}$ bewerten oder gar $\mathrm{zu}$ sanktionieren. Es geht vielmehr darum, erst einmal eine möglichst präzise und voraussetzungsarme Beschreibungssprache zur Verfügung zu stellen, mit deren Hilfe Gedichte mit autobiographisch inszenierenden Peritexten unabhängig von interpretationstheoretischen Vorannahmen zunächst analysiert werden können.

Orts- und Zeitangaben, die auf konkrete Stationen des Lebensweges der empirischen Verfasser verweisen oder zumindest die Disposition haben, als darauf referierend verstanden zu werden, finden sich tatsächlich auffällig häufig in den Peritexten von Gedichten. Albrecht von Hallers Schweizer Klassiker, das Alexandriner-Gedicht »Die Alpen«, das die verklärende Darstellung der ıreinen Sitten der Bergbewohner im Sinne Rousseaus vorwegnimmt, ist ein gutes Beispiel dafür, wie sich solche Peritexte von Textereignis zu Textereignis verändern könne. Heutigen Lesern begegnet es vermutlich nur noch in der seit der vierten Auflage von 1748 verbreiteten Fassung mit Angabe der Jahreszahl als Untertitel und einer autobiographischen Prosaeinleitung zwischen diesem Untertitel und der ersten Verszeile:

\begin{abstract}
Die Alpen
[Mart.] 1729

Dieses Gedicht [...] war die Frucht der großen Alpen-Reise, die ich An. 1728 mit dem jetzigen Herrn Canonico und Professor Geßner in Zürich getan hatte. Die starken Vorwürfe lagen mir lebhaft im Gedächtnis. [...] Ich wandte die Nebenstunden vieler Monate zu diesen wenigen Reimen an [...]. (Haller 2004, 3).
\end{abstract}

Bei seiner ersten Veröffentlichung 1732 in dem anonymen Bändchen Versuch Schweizerischer Gedichten [!] hatte dieser Text zunächst nur seinen Haupttitel und eine Nummerierung (»I. Die Alpen«).

25 Vor allem den vom 8. Juli 1828, also dem ersten Tag nach der Ankunft in Dornburg. Darin heißt es: »Früh in der Morgendämmerung das Tal und dessen aufsteigende Nebel gesehen. Bei Sonnenaufgang aufgestanden. Ganz reiner Himmel, schon zeitig steigende Wärme.« Es folgen einige knappe Angaben über die Tätigkeiten des Tages. Der Eintrag endet mit »Abends vollkommen klar. Heftiger Ostwind.« (Goethe 1993, 11). Die Nähe zu den Formulierungen des auf September datierten zweiten >Dornburger Gedichtes` ist unübersehbar. 


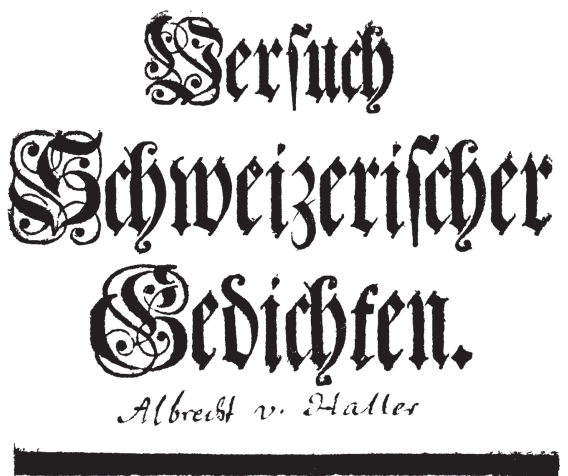

Stulta eft Clementia, cum tot ubique Vatibus occurras, perituræ parcere Chartæ. Iuvenal.
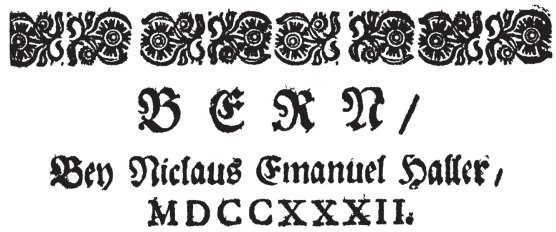

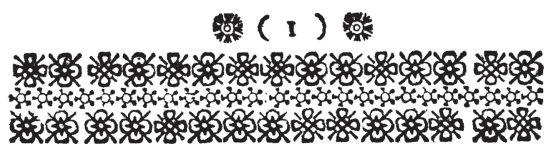

\section{Die $\mathfrak{A l p e n t}$}

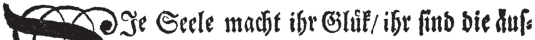
fern Sadhen /

(e) 6 Bur Ruft und sum 2 eroruß $/$ nar bic soe legenbeit :

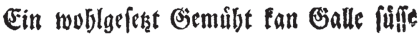
madjen /

Drein verwefinter Sinn auf nlles 2 sermuth fireut;

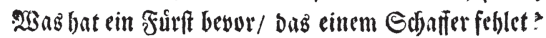

Der Scepter efelt ifm/ wie bem fein Scirten: Stab;

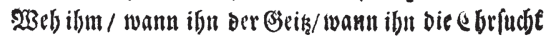
quâlet /

Die Schaar bie ifn bewadft/ bált ben 2 Berbrut nicfit $\mathfrak{a b}$ :

Der aber beffen Sinn gefezte Fube wieget /

Fragt er/ wann er entfoldifft/ ob cr auf $\subseteq$ difwanes lieget?

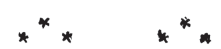

Sicgliulte guilone Zeit/ Du Erfftgeburt ber Jabren/

$\mathfrak{O}$ dẩ ber frimmel didf jo geitig meggerult!

Ricbt/ meil bie Zeiten noctb ein fthter Frúbling waren/

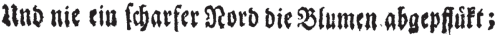

24

Abb. 3: [Albrecht von Haller:] Versuch Schweizerischer Gedichten. Bern 1732. Titelblatt und erste Seite des Gedichts »Die Alpen«, 1).

Im Zuge der Umarbeitungen seines Gedichtbandes für die zweite, dritte und schließlich vierte Auflage wurde die Inszenierung des Dichters aber offenbar immer wichtiger, bis hin zur quasi einen historischen Kommentar ${ }^{26}$ imitierenden Prosaeinleitung, die ab der vierten Auflage von 1748 darüber Auskunft gibt, wo, wann (1728) und bei welcher Gelegenheit dem Dichter die Idee zu dem Gedicht

26 Diese Nähe zu einem historischen Kommentar ist nicht zufällig ist: Zur gleichen Zeit erarbeiteten Hallers Freunde Bodmer und Breitinger in Zürich ihre gegen ahistorische Werk-Editionen gerichtete, damals völlig neuartige, historisch situierende und biographisch erläuternde OpitzAusgabe (vgl. Mahlmann-Bauer 2017). 
gekommen sei und wann sowie unter welchen Umständen er den darunter gedruckten Verstext schließlich ausgearbeitet habe.
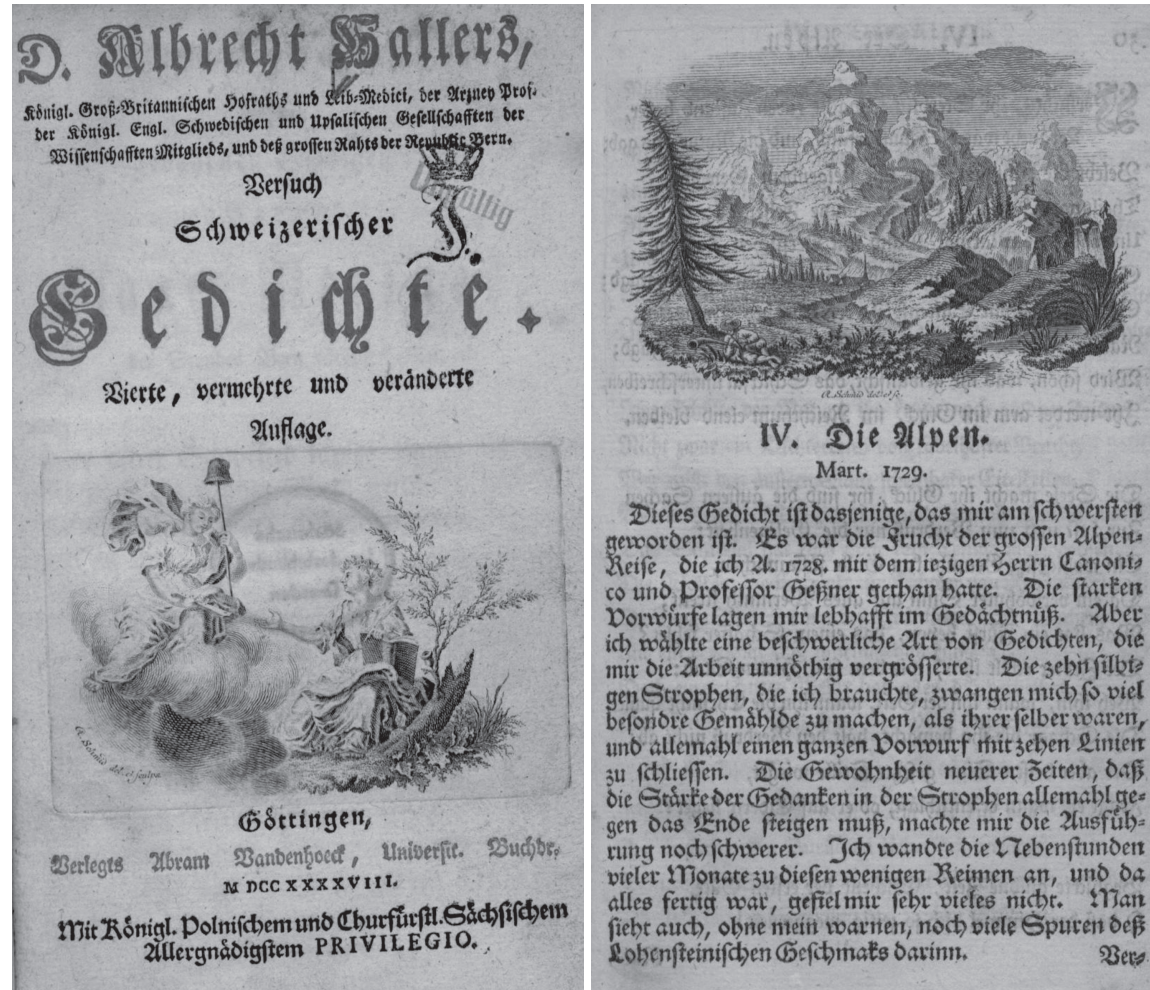

Diefes Gebicht ift oasjenige, oas mix an fot) twetftett

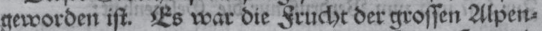
Reife, oie ich 2l. r728, mit bem iejigen Jertn Canonis co uno profeffor Gefner gethan batte. Die farken

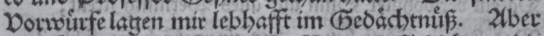

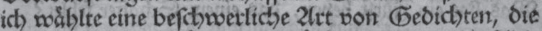
mir oie Zlibeit unnisthig vergrofferte. Die zebrif filbts gen Strophen, Dic id braud)te, swangen mich) fo viel befonore Gemabloe zu mact)en, als ibrer felber waren, unb allemableinen ganzen Dornourf frit zebsen Rinient

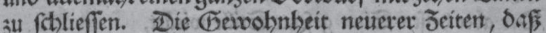

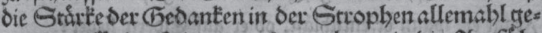

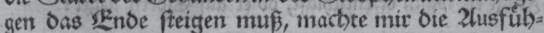

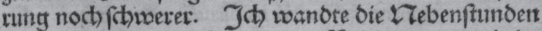
vielet IMonate zu Diefen wenigen Reimen an, uno $\partial a$ alles fertig war, gefielmir fehr vieles nict). IMan fiebt auth, obne nteit wartien, noch viele Sputen bef Lohenfteinifiden (s)eftomats batinn.

Abb. 4: Titelblatt von Hallers Versuch Schweizerischer Gedichte und erste Seite von »Die Alpen« in der verbesserten und erweiterten 4. Auflage, Göttingen 1748.

Eine biographische Erklärung für diese sich steigernde Intensität der dichterischen Selbstinszenierung lautet folgendermaßen: Bereits im Januar 1734, also noch vor Erscheinen der zweiten Auflage, war Hallers Bewerbung auf die Stelle des »dirigierenden Arztes« im Berner Inselspital von einem Mitglied des zuvor von Haller in mehreren satirischen Gedichten angegriffenen Berner Patriziats mit der Bemerkung abgelehnt worden: «Warum sollte dann der Dr. Haller wollen Spitalarzt werden, er ist ja ein Poet!« (Nachweis bei Siegrist 1967, 9). Dass diese Begründung ein Affront war, wird deutlich, wenn man bedenkt, dass `Poet` für aufgeklärte Dichter wie Haller im frühen achtzehnten Jahrhundert kein Beruf 
(wie etwa Spitalarzt) war, sondern dass Dichten als eine Beschäftigung praktisch tätiger Männer in ihren knappen Mußestunden angesehen wurde. ${ }^{27}$ Die Vermutung liegt also nahe, dass Haller die erste Auflage seiner Gedichte vor allem an ein lokales Publikum adressiert hatte, dann jedoch - wegen der Ablehnung in der Heimat, dem ohnehin rasch gelüfteten Inkognito, das ihn also nicht vor negativen Folgen seiner Spottlust geschützt hatte, und der überraschend großen Anerkennung seiner dichterischen Leistungen in Deutschland - sich ab der zweiten Auflage und von Auflage zu Auflage zunehmend überregional in einer Dreifachrolle inszenierte: als Schweizer (daher der Kupferstich einer Hochgebirgslandschaft in der vierten Auflage), als Dichter (der in Gedichtform, aber dennoch als er selbst spreche) und als Naturforscher (daher die Setzung zahlreicher botanisch erklärender Fußnoten in seinem langen Alpen-Gedicht). Nicht zuletzt durch die autobiographisch inszenierenden Peritexte hatten die adressantenmarkierten Gedichte von Auflage zu Auflage mehr die Disposition, die mentale Vorstellung einer Äußerungsinstanz zu triggern, die mit dem realen Textproduzenten identisch ist, der das im Gedichttext ausgesagte aus eigener Anschauung bzw. Erfahrung (im Sinne des naturwissenschaftlichen Kriteriums der Empirie) kennt.

Ähnlich komplex ist die Textgenese bei einem anderen, von der germanistischen Forschung sehr breit diskutierten (auch von Fabian Lampart im vorliegenden Band berücksichtigten) Gedicht: »Herrn Pauli Flemingi der Med. Doct. Grabschrifft / so er ihm selbst gemacht in Hamburg / den xxiix. Tag des Mertzens m. dc. xl. auff seinem Todtbette drey Tage vor seinem seel. Absterben« (Fleming 1646, 670).

Hier besteht zwar kein Zweifel daran, dass der Dichter sich um diese Zeit an diesem Ort aufgehalten hat und auch tatsächlich um dieses Datum herum in Hamburg gestorben ist. Doch zum einen lautete das behauptete Datum der Abfassung dieses Gedichtes in der Erstausgabe, einem Vorabdruck (»Prodromus«) von 1641, noch »20. Tag des Mertzen« (Fleming 1641, 50) - was schlicht ein Druckfehler (0 statt 8, in arabischen Ziffern leicht zu verwechseln) in der unter Zeitdruck entstandenen Ausgabe sein kann. Zum anderen jedoch ist fraglich, ob die Überschrift überhaupt vom (auf dem »Todtbette« liegenden) Dichter stammen könne, der zwar selbst Arzt, doch wohl kaum in der Lage war, seinen eigenen Todestag auf exakt drei Tage später vorhersehen zu können. Interessanterweise starb Fleming auch erst am 2. April 1640, also fünf, nicht »drey« Tage nach der im Gedichttitel gemachten Datumsangabe. Fraglich ist also, ob das Gedicht die Deutungsoption

27 So heißt etwa des Freiherrn von Canitz' erfolgreicher Gedichtband Neben-Stunden unterschiedener Gedichte (1700). 


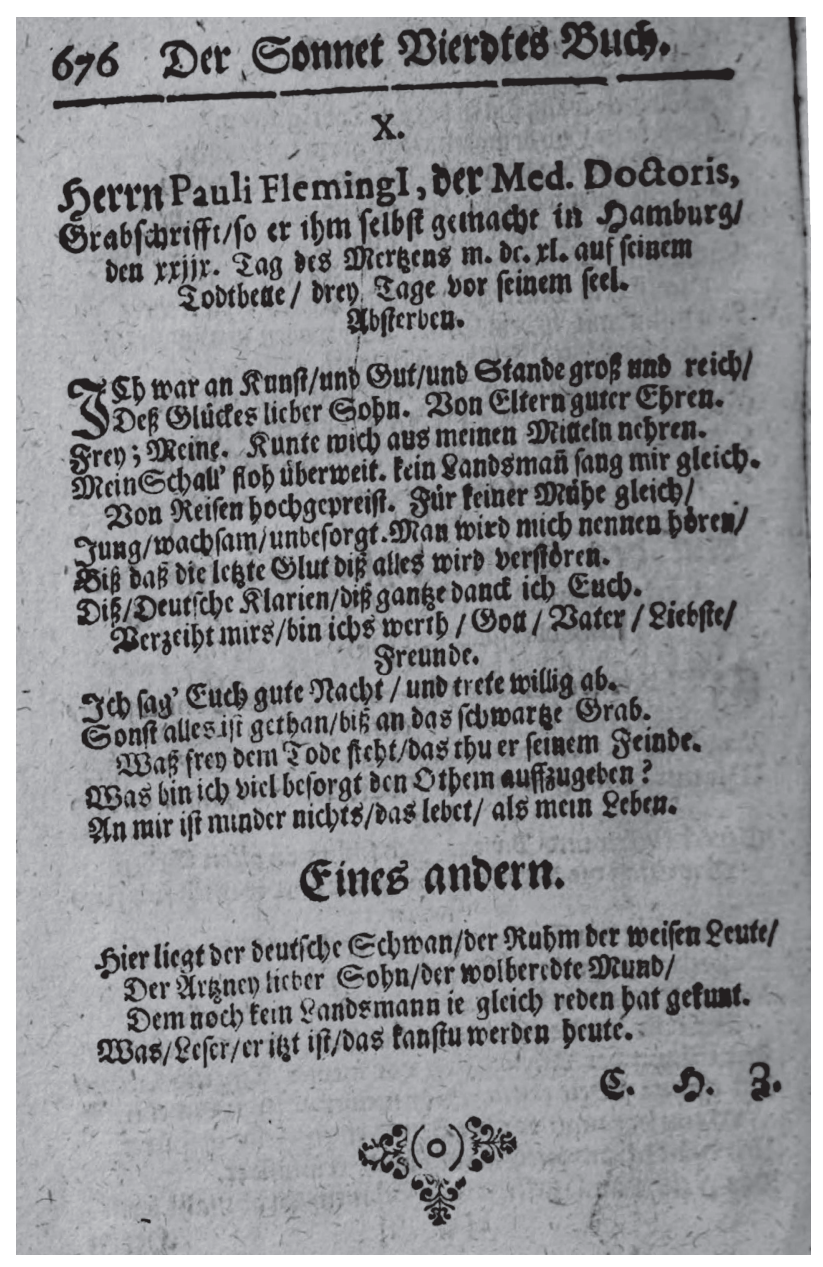

Abb. 5: Schluss von D. Paul Flemings Teütsche Poemata. Lübeck 1646, 670.

anbietet, dass hier >der Dichter selbst ` (oder zumindest `als er selbst`) spricht oder ob der Gedichttitel nur `Authentizität` suggerieren soll, wo in Wirklichkeit Rollenlyrik (das heißt eine zeitlich viel früher verfasste Stilübung Flemings zum damals verbreiteten Genre der Sterbegedichte, auf Englisch >Deathbed poetry<) vorliegt. Im Falle Flemings kann die Frage nach der Authentizität des Gedichttitels oder nach seiner nachträglichen Einfügung philologisch nicht beantwortet werden, da ein Original-Manuskript des Gedichtes nicht vorliegt. Dieses und andere Gedichte mit ähnlich autobiographisch authentifizierenden Peritexten jedoch nur als reine Vers-Haupttexte ohne Berücksichtigung der Peritexte zu betrachten und insofern 
ohne jede Rücksicht auf den Titel zu fragen »Wem kann ich die Sätze des Gedichtes als Äußerungen zuschreiben?«, hieße, die Texte radikal aus ihrem Kontext, ja sogar aus ihren Ko-Texten zu lösen und so wichtige Bedeutungsebenen abzuschneiden, wenn nicht gar, die Gedichte völlig misszuverstehen. ${ }^{28}$

Autobiographische Inszenierungen durch auf den Lebensweg der empirischen Autoren verweisende Orts- und Zeitangaben in den Peritexten von Gedichten können von fragwürdiger Glaubhaftigkeit sein (wie bei Fleming). Sie können in einer vergleichenden Analyse unterschiedlicher Textereignisse als nachträgliche Selbststilisierung erkennbar werden (wie bei Haller). Und sie können einem Gedicht, das auch ohne autobiographische Lesart für sich allein stehen könnte, eine völlig neue Deutungsrichtung hinzufügen (wie etwa bei Goethe). Nimmt man all diese Formen des Referierens auf außertextuelle Zeit und Orte des Lebenswegs der empirischen Verfasser zusammen, so funktionieren diese Orts- und Zeitangaben oft als `Faktualitätssignale`: Bei Angaben der Form 〉Ort, Komma, Datum` (etwa »Dornburg, 25. Aug. 1828« in der einem Brief an Marianne Willemer beigelegten Abschrift) handelt es sich um ein Formzitat aus explizit faktualen Textgattungen wie Vertrag, Brief, Tagebucheintrag oder Lebenslauf, deren Aufgabe es ist, die im Text getätigten Aussagen oder Vereinbarungen einer konkreten Person in der Realität zuzuweisen, an einem konkreten Ort, zu einem konkreten Zeitpunkt.

Offenbar lässt sich, was Wolfgang Kayser (1958) in seiner wirkmächtigen Studie Wer erzählt den Roman? als Postulat aufgestellt hat, dass nämlich der Erzähler eines Romans per definitionem nie der Autor sein könne, nicht ohne Abstriche auf Lyrik allgemein übertragen.

\section{Vorschlag eines Modells zur Integration von peritextuellen Referenzangeboten für die Deixis im Gedicht}

Für ein differenziertes Modell, das sowohl die von Vers-Haupttext als auch von den Peritexten angebotene Referenz für deiktische Ausdrücke im Gedicht berücksichtigen kann, bietet sich als Grundlage das von Semino (1997) aus der Textlinguistik in die Lyrikanalyse übertragene Beschreibungsmodel der `Text World Theory

28 Vgl. dazu auch Burdorf im vorliegenden Band. 
an. ${ }^{29}$ Mit diesem Modell lässt sich die »Binnenpragmatik« (Martínez 2002, 385) der deiktischen Ausdrücke zumindest für den Haupttext der Gedichten bereits jetzt sehr genau und unabhängig von fiktionstheoretischen Vorannahmen beschreiben: Jeder Text (also auch ein Gedicht, bei dem Textlichkeit vorliegt) hat die Disposition, dass Rezipienten seine Sätze als sprachliche Äußerungen verstehen und insofern die mentale Repräsentation einer Instanz, der diese Äußerungen zugeschrieben werden können, ausgelöst wird. Dieser >utterer muss in einem pragmatischen Kontext stehen, auf den sich zum Beispiel die deiktischen Ausdrücke in seiner Rede beziehen (der so genannte ১Verweisraum` der Deixis). Dieser mentale Vorstellungszusammenhang, der von der Gesamtheit des Textes im Rezipienten während der Rezeption getriggert wird, heißt in der Textlinguistik die stext world<. Dieser Terminus hat gegenüber dem in der Narratologie verbreiteter Konzept der sstory world ‘ den Vorteil, dass er nicht nur für Texte anwendbar ist, in denen eine Geschichte erzählt wird, und gegenüber dem in der Fiktionstheorie gebräuchlichen Konzept der >possible worldı oder >fictional world (so Lubomír Doležel und Marie-Laure Ryan), dass er nicht Fiktionalität zur Voraussetzung hat. Der Begriff der `Textwelt « ist weder an Narrativität noch Fiktionalität gekoppelt, sondern hängt allein davon ab, dass ein Text die Disposition hat, von Lesern als sprachliche Äußerung aufgefasst zu werden. In diesem zunächst rein textlinguistischen Sinne erzeugen also »auch Gedichte [...] Textwelten«, das heißt mentale Repräsentationen, die sich Rezipienten aufgrund konkreter Textmerkmale bilden. ${ }^{30}$ Ausnahmen bilden Texte, in denen »sprachliche Zeichen [...] primär visuelle oder akustische Eindrücke vermitteln«, das heißt nur noch mit ihnen » 'gemalt ` oder >musiziert « wird» (Anz 2007, 111). Solche Texte, die sich tatsächlich in ihrer spoetischen Funktion erschöpfen, bilden jedoch selbst in der Lyrik eher die Minderheit: »Die meisten literarischen Texte stellen etwas dar, genauer: « sie markieren ihren pragmatischen Ursprung und »evozieren Vorstellungen von etwas« (Anz 2007, 111). Und diese vom literarischen Text hervorgerufenen Vorstellungen der Rezipienten sind zu einem gewissen Maß durch die gleichen Kategorien bestimmt wie diejenigen Vorstellungen, die sich Menschen von ihrer realen Welt machen: nämlich durch die Kategorien von Raum, Zeit, >Gegenständen` (inklusive Personen) und Ereignissen, in die diese Gegenstände verwickelt werden (vgl. Anz 2007, 112). Der Begriff `Textwelt`

29 Zur Textwelttheorie in der Lyrikologie, vgl. grundsätzlich auch Rüdiger Zymners Beitrag zum JLT 2 (2017).

30 Bzw. bilden können, denn dass ein Text durch gewisse identifizierbare Merkmale die Disposition hat, eine mentale Vorstellung bei den Lesern auszulösen, heißt nicht, dass jeder einzelne Rezipient diese Vorstellung dann auch wirklich entwickelt (vgl. Müller 2003, 104) - hier spalten sich also empirische Leseforschung und text-orientierte Literaturwissenschaft. 
bezeichnet in diesem Sinne also den gesamten leserseitigen >Vorstellungszusammenhang`, der während der Lektüre eines Textes aufgrund von Textmerkmalen durch die Leser aktiv abgeleitet werden kann: die Worte eines Textes haben die Disposition, vom Rezipienten als Äußerungen einer Sprechinstanz aufgefasst zu werden, deren pragmatischer Kontext der Verweisraum der Deiktika im Text bildet (vgl. Semino 1997, 1-9).

Faktuale Texte werden so gelesen, dass dieser Verweisraum der Deiktika im Text prinzipiell im selben raumzeitlichen Kontinuum wie die außertextuelle Verweiswelt (die >Diskurswelt ${ }^{31}$ ) vorgestellt wird. Werden die Äußerungen im VersHaupttext jedoch als fiktionale Sätze rezipiert - zum Beispiel auf Grund von peritextuellen Elementen oder genre-typischen Markierungen, oder auch aufgrund der interpretationstheoretischen Vorannahme, dass Texte einer gewissen Textgruppenbildungen (z. B. Lyrik oder Literatur) immer fiktional seien -, so deutet der Leser sie als Aufforderung, sich die im Text gemachten Propositionen lediglich vorzustellen, ohne den einzelnen Aussagen Anspruch auf Referenzialisierbarkeit in der textexternen Diskurswelt zuzuschreiben: Der deiktische Verweisraum des Textes wäre somit als eine ontologisch von der eigenen Diskurswelt des Lesers (und der des empirischen Autors, denn diese beiden stehen ja in einem einzigen raumzeitlichen Kontinuum) getrennte Welt zu konzipieren (vgl. Klimek 2016).

Texte beschreiben Welten per se nur unvollständig, obwohl Leser prinzipiell (außer bei einigen Fällen phantastischer Literatur) davon ausgehen, dass diese Welten (seien sie nun fiktiv, seien sie real) ১vollständig`sind: Leser nehmen intuitiv an, dass auch für Umstände, die der Text nicht genau angibt, in der Textwelt etwas der Fall sein muss. Zu den Unbestimmtheitsstellen im Text, die die Leser bei ihrer Lektüre auf die ein oder andere Weise automatisch auffüllen oder sich diese zumindest auffüllbar denken, gehören diejenigen Ausdrücke, die nur im pragmatischen Vollzug verstanden werden können, das heißt die Deiktika. Um zu verstehen, auf wen oder was sich kontextsensitive Ausdrücke wie >ich`, ’hier`, ১jetzt`, >oben « etc. in Texten beziehen, konzeptualisieren Leser eine Sprechinstanz als Textproduzent im Zentrum der Textwelt, um welches herum sich das deiktische Feld konstituiert und auf die sich alle Deiktika im Text beziehen. Die Deiktika, die bei der mentalen Konstruktion der Welt eine Rolle spielen, beschränken sich jedoch keineswegs auf die raumzeitlichen Ausdrücke, sondern umfassen auch alle Bereiche der $>$ perceptual deixis $\iota^{32}$ und $>$ relational deixis ${ }^{33}$.

31 Diskurs hier im linguistischen Sinne, nicht im auf Foucault zurückgehenden.

32 Vgl. Stockwell (2002, 45): »expressions concerning the perceptive participants in the text, including pronouns [...], definite articles, [...] mental states«.

33 Vgl. Stockwell (2002, 45-46): »expressions that encode the social viewpoint and relative 
Haben wir es mit einem Ich-Gedicht ohne offenkundige Rollenfiktion zu tun, oder auch mit einem Gedicht ohne jegliche Verwendung von Personalpronomina, die auf das deiktische Zentrum zurückschließen lassen, also mit der vom Gedichttext angestoßenen Vorstellung einer »nichtmarkierte[n] Sprechinstanz« (vgl. Fricke und Stocker 2000), so ist das Zentrum des deiktischen Feldes semantisch unterdeterminiert. Dies gilt etwa auch für den Haupt-Verstext von Goethes zweitem Dornburger Gedicht. Hier taucht zwar in der dritten Strophe ein direkt angesprochenes »Du « auf, doch wird dieser Adressat nicht in seiner tatsächlichen aktuellen Lage angesprochen, sondern ihm wird mit Hilfe einer »Wenn-dann«Konstruktion ein vielleicht kausaler, vielleicht auch nur hypothetischer Zusammenhang geschildert. Alle weiteren Informationen über die Textwelt, von den im engeren Sinne deiktischen Ausdrücken im Gedicht (etwa die Angabe der Tageszeit, »[f]rüh«) bis zu den diversen Wettererscheinungen (»Nebelschleier[...] «, »Ostwind«, »blaue Sonnenbahn«) beziehen sich also nur auf diese suggerierte Situation, in die sich das angesprochene Du hineindenken soll. Zudem wäre es für die Lyrik durchaus nicht unüblich, dass der Adressat mit dem Adressanten identisch ist, es sich also bei der im Rezipienten getriggerten Vorstellung um die eines Selbstgesprächs handeln könnte. Auskunft über die eigentliche Textwelt geben einzig die >deiktischen Ausdrücke ‘ im weiteren Sinne (`social deixis` und >compositional deixis`, vgl. Stockwell 2013, 271), an denen man ablesen kann, dass der im Gedicht markierte Adressant über einen elaborierten Code sowie eine differenzierte Grammatik verfügt und insofern die Vorstellung einer sehr gebildeten Person als Äußerungsinstanz des Gedichtes triggert. Darüber hinaus bleibt die vom Text gelieferte Information über diese Äußerungsinstanz jedoch sehr vage.

Nachdem man mit Hilfe einer sorgfältigen Analyse der gedichtinternen Deixis die Textwelt und ihr deiktisches Zentrum möglichst genau bestimmt hat (im Falle Goethes etwa als eine gebildete, aber ansonsten semantisch unterdeterminierte `Äußerungsinstanzı ohne erkennbare Rollenfiktion, die sich an irgendeine Form von Adressat wendet, eventuell in einem Selbstgespräch), sollen nun in einem zweiten Schritt auch die Peritexte berücksichtigt werden. Im Falle von Goethes Gedicht wäre da also zunächst der Titel, »Dornburg, Septbr. 1828«.

situations of authors, narrators, characters, and readers, including [...] expressions of point of view and focalisation «. - Laut Stockwell $(2002,46)$ gehören sogar `textual deixis` (»expressions that foreground the textuality of text, including explicit ssignposting such as chapter titles and paragraphing «) und »compositional deixis « (»aspects of the text that manifest the generic type or literary conventions available to readers with the appropriate literary competence«) dazu. 
Orts- und Zeitangaben, die nachweisbar auf Stationen des Lebensweges des empirischen Autors verweisen, können drei verschiedene Funktionen haben:

a) Man kann sie in der Rezeption als auf den pragmatischen Vollzug der Äußerungen, als die man den Haupt-Verstext deutet, auffassen: Wo und wann werden die folgenden Äußerungen getätigt?

b) Man kann sie auf die Inhaltsebene des Vershaupttextes beziehen: An welchem Ort und zu welcher Zeit findet das im Verstext Dargestellte statt?

c) Oder man kann sie auf die schriftliche Produktion des Gedichtes beziehen: Wo und wann hat der reale Autor dieses Gedicht niedergeschrieben?

Hempfer $(2015,34)$ sieht das prototypisch Lyrische unter anderem durch ein Zusammenfallen von a) Äußerung des Gedichts und b) im Gedicht dargestellter Situation charakterisiert. Doch für die große Menge der nicht in diesem Sinne prototypischen Lyrik gilt es, a) und b) durchaus auseinander zu halten. Im Falle Goethes wird von der Forschung einhellig davon ausgegangen, dass (zumindest auch) c) der Fall ist: Das Gedicht scheint tatsächlich während Goethes Aufenthalt auf den Dornburger Schlössern im Herbst 1828 entstanden zu sein (vgl. Eibl 1988, 1218). ${ }^{34}$ Auch a) könnte zusätzlich zu c) der Fall sein. Dann müsste man sich die Äußerung der vom Text ausgelöste Vorstellung einer Äußerungsinstanz als in Dornburg im September 1828 getätigt vorstellen. Die Peritextwelt liefert also eine Art Folie, die man nicht nur über die eigene Vorstellung von der Schreibsituation legen kann, ${ }^{35}$ sondern auch über die Äußerungssituation im Text. Da ansonsten aber wenig über das Zentrum des deiktischen Feldes aus dem Text heraus ableitbar ist, bleibt b) als mögliche neue Verständnisquelle: Wenn man sich vorstellt, dass der vom Adressanten im Gedicht - sei es kausal, sei es potential - beschriebene Wenn-dann-Zusammenhang in Dornburg im September 1828 bestand, dann kann man die erwähnten Wetterphänomene der frühherbstlichen Jahreszeit im Saaletal zuordnen. Aus einer konkreten Wetterbeobachtung wäre dann ein allgemeingültiges Gesetzt abgeleitet worden. Man könnte sogar im fraglichen Monat nach Dornburg reisen und sich - bei entsprechender Wetterlage - im von Goethe bewohnten Zimmer aufhalten und empirisch klären, ob man von dessen Fenstern aus den Sonnenauf- und auch den Sonnenuntergang beobachten kann. ${ }^{36}$

34 Dies ist natürlich nur von Bedeutung, falls man denn eine interpretationstheoretische Grundhaltung vertritt, in der empirischen Autoren und ihren Schreibsituationen irgendeine Bedeutung zukommt.

35 So man sich denn solche Vorstellungen überhaupt bilden will, vgl. oben.

36 Detering (2009) argumentiert nämlich auf Grundlage eines Parallelstellenvergleichs, dass die »rötlich scheidend[e]«»Sonne« im vorletzten Vers immer noch auf den Sonnenaufgang bezogen werden müsse. 
Unabhängig davon, ob der einzelne Literaturwissenschaftler ein solch praktisches Vorgehen für gerechtfertigt oder absurd hält, kann man mit Hilfe der lyrikologischen Beschreibungskategorien immerhin das Zustandekommen solchen Verhaltens als Wirkungsdisposition des Gedichttextes beschreiben: „Dornburg « ist Teil sowohl der Diskurswelt des Rezipienten als auch der des empirischen Autors (da sich beide im selben raumzeitlichen Kontinuum befinden). Verbunden mit der Zeitangabe wird ein Punkt in diesem Kontinuum benannt, an dem sich der empirische Autor nachweislich befunden hat. Er ist der reale Urheber des lyrischen Schriftzeichengebildes. Dieses Schriftzeichengebilde markiert einen Adressanten (als pragmatischen Ursprung des Gedichts im Gedicht), der sich als das Zentrum des vom Text aufgespannten deiktischen Feldes vorstellen lässt, das heißt als Äußerungsinstanz. Der Peritext spannt eine Peritextwelt auf, die mit der Diskurswelt durch die Referenz zusammenfällt, und bietet somit auch eine mögliche Referenz für die Aussagen im Haupt-Verstext an. Autobiographisch inszenierende Peritexte haben die Wirkungsdisposition, eine solche Referenz-Übertragung beim Rezipienten auszulösen - sofern dieser Mechanismus nicht durch Vorannahmen des Rezipienten (etwa die Prämisse, Lyrik könne - als Kunst und Literatur - nie auf Wirklichkeit referieren, oder aber durch einen fiktionalen Rahmen, wie die womöglich fiktionale Rahmenerzählung in Dantes Vita Nova) blockiert ist. Soweit die Analyse. Ob man für dieses Gedicht nun konkret die Lesart a) und b) in Anschlag bringen will, bleibt darüber hinaus eine Frage der Interpretation. ${ }^{37}$

Mit Burdorfs (2015, 194-201) Modell konnte man bisher aussagen, dass der empirische Autor Goethe mit diesem Gedicht ein `Textsubjekt` (entspricht etwa dem in der Narratologie umstrittenen Konzept des simplied author C. Booth) geschaffen hat, welches im Gedicht ein rartikuliertes Ich « sich an einen gedichtinternen `Zuhörer` wenden lässt, um einen vom `Textsubjekt` sintendierten Leser` (also ein rezeptionsästhetisches Konstrukt) zu erreichen. Dieses solchermaßen beschreibbare Gedicht wird wieder von realen Lesern rezipiert. Mit Hilfe der Textweltanalyse kann man dieses sartikulierte Ich nun jedoch 1.) als vom Text getriggertes mentales Konstrukt der Leser näher bestimmen, das hervorzurufen konkrete Textmerkmale die Disposition haben. Und 2.) kann man sodann mit Hilfe der Peritextweltanalyse Argumente für die Plausibilität der Annahmen a), b) und/oder c) finden.

37 Für ein »historisch-hermeneutisch orientiertes Textverstehen« und eine »Interpretation" (Martínez 2002, 385) ist natürlich die genaue Aufschlüsselung der Referenz dieser Orts- und Zeitangaben notwendig - wenn man denn historisch-hermeneutisch arbeiten will. Für die Berechtigung einer solchen Interpretation kann die hier vorgeschlagene, textlinguistisch fundierte Peritextwelt-Analyse jedoch ein weiteres stützendes Argument liefern. 
Im Falle des oben genannten Haller-Beispiels handelt es sich also um ein 1732 zum ersten Mal ohne autobiographische Peritexte publiziertes Gedicht mit dem Titel »Die Alpen«. Ab der vierten Auflage gibt der Prosa-Vortext an, dass b) die im Gedicht besprochene Alpenreise im Jahre 1728 stattgefunden habe und die Produktion des Gedichtes c) ein langwieriger Akt voller Überarbeitungen gewesen sei, der sich über mehrere Monate hingezogen habe. Was aber gibt der in der dritten Auflage eingefügte Untertitel »Mart. 1729« dann an? Die Niederschrift kann es ja nicht sein, denn die war ja nicht binnen eines Monats abgeschlossen. Es bleibt also nur a). Der Monat März 1729, also ein Zeitpunkt nach Beendigung der im Gedicht dargestellten sommerlichen Reise, soll als der Moment vorgestellt werden, an dem der vom Gedicht markierte Adressant seine Äußerung tätigt. Das bedeutet, dass wir uns die als Äußerungen vorgestellten Schriftzeichen als im gleichen Raumzeitkontinuum getätigt vorstellen können, in dem auch wir leben (und in dem auch Haller lebte), dass diese Äußerungsinstanz aber sehr wohl von Haller verschieden ist, da Haller ja selbst behauptet, am Text gefeilt zu haben und zwar länger als einen einzigen Monat.

Ein letztes Beispiel soll noch einmal illustrieren, dass das Einbeziehen autobiographischer Peritexte in die Analyse von »Ich-Gedichten ohne Rollenfiktion « (Fricke und Stocker 2000) mitnichten zu ınaivem Biographismus führen muss. Erneut wähle ich einen Text des frühen achtzehnten Jahrhunderts, in dem kleine Abwandlungen der Orts- und Zeitangaben in den Peritexten die Wirkungsdisposition des Textes völlig verändern - obwohl die »Binnenpragmatik« (vgl. Martínez 2002, 385) des im Vershaupttext angelegten deiktischen Feldes sich nicht verändert. Es handelt sich um das auf eine reale Katastrophe Bezug nehmende, Ende 1736 als Separat-Druck bei Johann Heinrich Nonnen in Erfurt publizierte Brandgedicht »Das am 21= und 22ten October 1736 unter Gluth und Flammen ächzende Erfurt, vorgestellt von Sidonia Hedwig Zäunemannin« (Zäunemann 1736). Im Gedicht gibt es einen nicht näher markierten Adressanten, der die Vorstellung einer Äußerungsinstanz triggert, die eine kommentierende, wertende, emotional Anteil nehmende Beschreibung der Ereignisse jener Brandkatastrophe liefert. Solche poetischen Darstellungen von großen Katastrophen waren im frühen achtzehnten Jahrhundert durchaus üblich. Die Orts- und Zeitangaben in den Titeln der Gedichte dieses Genres bezogen sich b) auf das im Gedicht Dargestellte (hier also das Feuer), über dessen solche meist auf Flugblättern verteilten Gedichte informieren sollten.

Als die Zäunemannin das besagte Gedicht jedoch zwei Jahre später in ihre Gedichtsammlung Poetische Rosen in Knospen aufnahm, lautete die Zeitangabe im Titel leicht variiert: »Erfurt. Den 21ten Oct. 1736« (Zäunemann 1738, 537). Aus der Angabe von zwei Tagen, während denen die im Gedicht dargestellte Begebenheit sich ereignete, wird so die Orts- und Datumsangabe, wie sie in Briefen üblich ist, 
um a) Ort und Zeitpunkt der sprachlichen Äußerungen näher zu bestimmen. Aus der rückschauenden Beschreibung und Reflexion über eine Feuersbrunst wird so eine lyrische Live-Berichterstattung von der Grenze zwischen Leben und Tod: Die Dichterin inszeniert sich selbst in der zweiten Fassung als diejenige, die beim Brand dabei ist und - im Sinne Hempfers - simultan zum Geschehen spricht. Die Tatsache, dass man aus den verschiedenen Textzeugen eine Arbeit an der Selbstinszenierung als Äußerungsinstanz des Gedichtes ablesen kann, widerlegt jeden >Naivitätsvorwurfı: Es handelt sich bei der ıautobiographischen Lesartı, die solche Peritexte anregen können, nicht um Fehllektüren, sondern um gezielt kalkulierte Wirkungen bzw. dem Text eigene, an konkreten Textmerkmalen festzumachende Wirkungsdispositionen.

Diese Veränderung der Peritexte als Fiktionalitätssignal für den ganzen Text zu werten, halte ich für historisch nicht angemessen. Natürlich haben auch Zeitgenossen nicht angenommen, dass die Zäunemannin mit Papier und Feder in der Hand durch das brennende Erfurt gelaufen sei. Aber man kannte sie als Erfurterin. Die Rolle der Augenzeugin ist also plausibel. Sie wird hier lediglich besonders hervorgehoben. Es handelt sich daher um eine rhetorische Zuspitzung der Performativität in einer Textgattung, in der Sinn ohnehin durch ein Ineinander von Aufrichtigkeitsanspruch und Konventionen der rhetorischen Stilisierung und vielen weiteren Arten des >making specialı generiert wird. Der pragmatische Status der Rede (das heißt sein Anspruch auf Faktualität, auch wenn dieser ein nur simulierter sein könnte) ist davon nicht betroffen.

\section{Thesen}

Aus diesen Beispielen und konzeptionellen Überlegungen möchte ich abschließend folgende Thesen extrahieren:

1. Orts- und Zeitangaben in den Peritexten von Ich-Gedichten ohne offenkundige Rollenfiktion können die Referenz für das an sich semantisch unterdeterminierte Zentrum des deiktischen Feldes im Gedichttextes anbieten.

2. Als Formzitate aus autorfaktualen Textsorten können sie unter bestimmten Bedingungen die Wirkungsdisposition haben, als Faktualitätssignale wahrgenommen zu werden (sofern diese Option nicht durch anderweitige Lektürevorgaben wie historische Genrekonventionen oder durch interpretationstheoretische Vorannahmen ausgeschlossen ist).

3. Der Anspruch auf Faktualität dieser peritextuellen Orts- und Zeitangaben kann, muss aber nicht, ein simulierter sein. 
4. Im Rahmen einer historisch-hermeneutischen Gedichtinterpretation ist vorgängig zu klären, auf welches Textereignis man sich beziehen will, wie dort die Referenz anbietenden Peritexte genau lauteten und ob es in der Textgenese gegebenenfalls auch andere Angebote der Referenz in anderen Peritexten gab.

5. Da lyrische Sprachzeichengebilde wie alle Sprachzeichengebilde aus arbiträren Zeichen bestehen, das heißt ihre Bedeutung durch Konventionen geregelt ist, sind für eine historisch-hermeneutische Gedichtinterpretation zudem die verbindlichen, literargeschichtlich bedingten Genre-Konventionen zu rekonstruieren, die zur Zeit der Text-Entstehung sowie der jeweiligen TextereignisEntstehung als verbindlich galten.

Fiktionalität der Lyrik zu postulieren hieße (zumindest für die Frühe Neuzeit und die Frühmoderne, und nur Beispiele aus diesem Zeitraum wurden im vorliegenden Aufsatz behandelt), die Möglichkeit eines historisch-hermeneutischen Verständnisses der sehr komplexen und historisch wandelbaren Beziehungen zwischen Lyrik und Autobiographik von vorne herein $\mathrm{zu}$ beschneiden. Legt man ein institutionelles Verständnis von Fiktionalität zugrunde, so ist das Konzept zudem ohnehin nicht transhistorisch stabil. Dieser Beitrag versteht sich insofern als ein Plädoyer dafür, die Frage nach den Anteilen von Fiktionalität oder Faktualität von Lyrik für jeden Einzelfall im Kontext der zur Entstehungszeit verbindlichen GenreKonventionen und niemals unter Ausklammerung der das Gedicht rahmenden (unter Umständen autobiographisch inszenierenden) Peritexte zu behandeln. Die Antwort auf die Frage »Wer spricht im Gedicht?«, die sich auf die vom Text beim Rezipienten angestoßene Vorstellung einer Äußerungsinstanz bezieht, kann daher durchaus lauten: `Es spricht der Dichter selbst.` Auch wenn dieses ıSprechen als er selbst « Lügen, Übertreibungen oder Stilisierungen enthalten mag und vielleicht selbst schon wieder nur eine Rolle ist. ${ }^{38}$

38 Die Recherchen zu diesem Aufsatz habe ich mit Hilfe eines Residenzstipendiums der Klassik Stiftung Weimar im Sommer 2015 und eines Stipendiums des Forschungsverbunds Marbach Weimar Wolfenbüttel im Frühjahr 2017 in der Herzog August Bibliothek durchgeführt. Mein herzlicher Dank gilt Herrn Prof. Dr. Thorsten Valk vom Stabsreferat Forschung und Bildung in Weimar sowie dem stellvertretenden Leiter der Abteilung Forschungsplanung und Forschungsprojekte Dr. Hole Rößler in Wolfenbüttel für die Erteilung dieser überaus hilfreichen Förderungen. 


\section{Literaturverzeichnis}

Anz, Thomas. »Textwelten«. Handbuch Literaturwissenschaft. 1. Bd. Gegenstände und Grundbegriffe. Hg. Thomas Anz. Stuttgart: Metzler, 2007. 111-130.

Blasing, Mutlu Konuk. Lyric Poetry. The Pain and the Pleasure of Words. Princeton: Princeton Univ. Press, 2006.

Blödorn, Andreas, Daniela Langer und Michael Scheffel. »Einleitung: Stimmen - im Text?«. Stimme(n) im Text. Narratologische Positionsbestimmungen. Hg. Andreas Blödorn, Daniela Langer und Michael Scheffel. Berlin: de Gruyter, 2006.1-7.

Borkowski, Jan und Simone Winko. „Wer spricht das Gedicht? Noch einmal zum Begriff slyrisches Ich، und zu seinen Ersetzungsvorschlägen «. Lyrische Narrationen - narrative Lyrik. Gattungsinterferenzen in der mittelalterlichen Literatur. Hg. Hartmut Bleumer und Caroline Emmelius. Berlin: de Gruyter, 2011. 43-77.

Boutière, Jean und Alexander H. Schutz (Hg.). Biographies des troubadours: textes provençaux des XIIle et XIVe siècle. Paris: Nizet, 1964.

Braungart, Georg. »Barocke Grabschriften: Zu Begriff und Typologie». In: Studien zur Literatur des 17. Jahrhunderts. Hans Feger (Hg.). Amsterdam: Rodopi, 1997. 425-487.

Burdorf, Dieter. Einführung in die Gedichtanalyse [1995]. 3. Aufl. Stuttgart: Metzler, 2015.

Canitz, Friedrich Rudolph Ludwig Freiherr von, anonym. Neben-Stunden Unterschiedener Gedichte. Berlin: Johann Michael Rudiger, 1700.

Culler, Jonathan. Theory of the Lyric. Cambridge, MA: Harvard Univ. Press, 2015.

Dante. Vita Nova. Italienisch - Deutsch. Übersetzt von Hanneliese Hinderberger. Basel: Schwabe, 1947.

Detering, Heinrich. »Farbenlehre und Lichtkult. Goethes Dornburger Gedichte«. Jahrbuch der Akademie der Wissenschaften zu Göttingen (2009): 192-204.

Deutscher Musenalmanach für das Jahr 1833. Hg. A. von Chamisso und G. Schwab 4 (1833). Leipzig: Weidmannsche Buchhandlung, 1832.

Eibl, Karl. »Kommentar«. Johann Wolfgang Goethe. Sämtliche Werke. I,1l: Gedichte 1800-1832. Hg. Karl Eibl. Frankfurt a. M.: Deutscher Klassiker Verlag, 1988. 873-1365.

Fischer, Carolin. Der poetische Pakt. Rolle und Funktion des poetischen Ich in der Liebeslyrik bei Ovid, Petrarca, Ronsard, Shakespeare und Baudelaire. Heidelberg: Winter, 2007.

Fleming, Paul. D. Paul Flemings Poetischer Gedichten So nach seinem Tode haben sollen herauß gegeben werden. Prodromus. Hamburg: Gunderman, 1641.

Fleming, Paul. D. Paul Flemings Teütsche Poemata. Lübeck: Jauch, 1646.

Fricke, Harald und Peter Stocker. "Lyrisches Ich«. Reallexikon der deutschen Literaturwissenschaft. 2. Bd. Hg. Harald Fricke, Klaus Grubmüller, Jan-Dirk Müller und Klaus Weimar. Berlin: de Gruyter, 2000. 509-511.

Gabriel, Gottfried. »Fiktion«. Reallexikon der deutschen Literaturwissenschaft. 1. Bd. Hg. Harald Fricke, Klaus Grubmüller, Jan-Dirk Müller und Klaus Weimar. Berlin: de Gruyter, 1997. 594-598.

Genette, Gérard. „Discours du récit«. Gérard Genette. Figures III. Paris: Seuil, 1972. 67-282.

(Dt.: Gérard Genette. Die Erzählung. Übersetzt von Andreas Knop. Hg. Jochen Vogt.

München: Fink, 1994.)

Genette, Gérard. Paratexte. Das Buch vom Beiwerk des Buches [1987]. Übersetzt von Dieter Hornig. Frankfurt a. M.: Suhrkamp, 2001.

Genette, Gérard. Métalepse. De la figure à la fiction. Paris: Seuil, 2004. 
Goethe, Johann Wolfgang. Goethe's Werke. Vollständige Ausgabe letzter Hand. 47. Bd. Stuttgart, Tübingen: J. G. Cotta, 1833.

Goethe, Johann Wolfgang. Sämtliche Werke, Briefe, Tagebücher und Gespräche. 40 Bde. II. Abteilung; 11. Bd. Briefe, Tagebücher und Gespräche von 1823 bis zu Goethes Tod. Teil II. Hg. Horst Fleig. Frankfurt a. M.: Deutscher Klassiker Verlag, 1993.

Greenblatt, Stephen. Renaissance Self-Fashioning. From More to Shakespeare. Chicago: Univ. of Chicago Press, 1980.

[Haller, Albrecht von; anonym]. Versuch Schweizerischer Gedichten. Bern: Nicolaus Emanuel Haller, 1732.

Haller, Albrecht von. Versuch Schweizerischer Gedichte. 4. Aufl. Göttingen: Abram Vandenhoeck, 1748.

Haller, Albrecht von. Die Alpen und andere Gedichte [1965]. Auswahl und Nachwort von Adalbert Elschenbroich. Stuttgart: Reclam, 2004.

Hamburger, Käte. Die Logik der Dichtung [1957]. 2. Aufl. Stuttgart: Klett, 1968.

Hempfer, Klaus W., Lyrik. Skizze einer systematischen Theorie. Stuttgart: Steiner, 2014.

Hillebrandt, Claudia. »Author and Narrator in Lyric Poetry«. Author and Narrator. Hg. Dorothee Birke und Tilmann Köppe. Berlin: de Gruyter, 2015. 213-233.

Hühn, Peter und Jörg Schönert. »Einleitung: Theorie und Methodologie narratologischer Lyrikanalyse«. Lyrik und Narratologie. Textanalysen zu deutschsprachigen Gedichten vom 16. bis zum 20. Jahrhundert. Hg. Jörg Schönert, Peter Hühn und Malte Stein. Berlin: de Gruyter, 2007. 1-17.

Kablitz, Andreas. »Fiktion und Bedeutung. Dantes Vita nova und die Tradition der volkssprachlichen Minnelyrik«. Fiktion und Fiktionalität in den Literaturen des Mittelalters. Hg. Ursula Peters und Rainer Warning. München: Fink, 2009. 339-362.

Kayser, Wolfgang. »Wer erzählt den Roman?« [1957]. Wolfgang Kayser. Die Vortragsreise. Studien zur Literatur. Bern: Francke, 1958. 82-101.

Kindt, Tom und Hans-Harald Müller. »Was war eigentlich der Biographismus - und was ist aus inm geworden?« Autorschaft. Positionen und Revisionen. Hg. Heinrich Detering. Stuttgart: Metzler, 2002. 355-375.

Klimek, Sonja. »Textwelten der Lyrik - Spielräume der Interpretation. Am Beispiel von Goethes >Dem aufgehenden Vollmonde«. Spielräume und Raumspiele in der Literatur. Hg. Elisabeth Heyne und Julia Dettke. Würzburg: Königshausen \& Neumann, 2016. 85-103.

Klimek, Sonja. »Functions of figurativity for the narrative in lyric poetry. With a study of English and German poetic epitaphs from the 17th century«. Language and Literature 22.2 (2013): 219-231.

Köppe, Tilmann und Tom Kindt. Erzähltheorie. Eine Einführung. Stuttgart: Reclam, 2014.

Köppe, Tilmann und Jan Stühring. "Against Pan-Narrator Theories«. Journal of Literary Semantics 40 (2011): 59-80.

Lamping, Dieter. Das lyrische Gedicht. Definitionen zu Theorie und Geschichte der Gattung [1989]. 3. Aufl. Göttingen: Vandenhoeck \& Ruprecht, 2000.

Lejeune, Philippe. Der autobiographische Pakt. Aus dem Französischen von Wolfram Bayer und Dieter Hornig. Frankfurt a. M.: Suhrkamp, 1994.

Mahlmann-Bauer, Barbara. »Die Opitz-Edition Bodmers und Breitingers (1745)«. Zeitschrift für Germanistik, 27.1 (2017): 53-68.

Martínez, Matías. »Das lyrische Ich. Verteidigung eines umstrittenen Begriffs«. Autorschaft: Positionen und Revisionen. Hg. Heinrich Detering. Stuttgart: Metzler, 2002. 376-389. 
Müller, Wolfgang G. Das lyrische Ich. Erscheinungsformen gattungseigentümlicher AutorSubjektivität in der englischen Lyrik. Heidelberg: Winter, 1979.

Müller, Ralph. Theorie der Pointe. Paderborn: mentis, 2003.

Müller, Wolfgang G. „Das lyrische Ich«. Handbuch Lyrik. Theorie, Analyse, Geschichte. Hg. Dieter Lamping. Stuttgart: Metzler, 2011. 56-58.

Müller-Zettelmann, Eva. »Lyrik und Narratologie«. Erzähltheorie transgenerisch, intermedial, interdisziplinär. Hg. Vera Nünning und Ansgar Nünning. Trier: Wissenschaftlicher Verlag, 2002. 129-153.

Petzold, Jochen. Sprechsituationen lyrischer Dichtung. Ein Beitrag zur Gattungstypologie. Würzburg: Königshausen \& Neumann, 2012.

Rabaté, Dominique. Figures du sujet lyrique. Paris: Presses universitaires de France, 1996. Rabaté, Dominique, Joëlle de Sermet und Yves Vadé (Hg.). Le Sujet lyrique en question. Bordeaux: Presses Universitaires de Bordeaux, 1996.

Ronsard, Pierre de. „Sonnets et madrigals pour Astrée« [1578]. Ders.: Amours pour Marie / Amoren für Marie. Hg. und Übersetzung von Carolin Fischer. Berlin: Elfenbein, 2010.

Schiedermair, Simone. ILyrisches Ich` und sprachliches sich`. Literarische Funktionen der Deixis. München: Judicium, 2004.

Schöne, Albrecht. Goethes Farbentheologie. München: Beck, 1979.

Schönert, Jörg. Empirischer Autor, Impliziter Autor und Lyrisches Ich [1999], mit einem Nachtrag 2004. http://www.icn.uni-hamburg.de/webfm_send/40 (10. September 2016).

Segebrecht, Wulf. „Steh, Leser, still! Prolegomena zu einer situationsbezogenen Poetik der Lyrik, entwickelt am Beispiel von poetischen Grabschriften und Grabschriftenvorschlägen in Leichencarmina des 17. und 18. Jahrhunderts«. DVJS 52 (1978): 430-468.

Semino, Elena. Language and World Creation in Poems and Other Texts. London: Longman, 1997. Siegrist, Christoph: Albrecht von Haller. Stuttgart: Metzler, 1967.

Stockwell, Peter. Cognitive Poetics: An Introduction. London: Routledge, 2002.

Stockwell, Peter. »The positioned reader«. Language and Literature 22.3 (2013): 263-277.

Susman, Margarete. Das Wesen der modernen deutschen Lyrik. Stuttgart: Strecker \& Schröder, 1910.

Zäunemann, Sidonia Hedwig. Das am 21- und 22ten October 1736 unter Gluth und Flammen ächzende Erfurt. 2. Aufl. Erfurt: Nonne, [1736]. [Wolfenbüttel HAB: Gem 1649.]

Zäunemann, Sidonia Hedwig. Sidonien Hedwig Zäunemannin, Kayserlich gekrönter Poetin, Poetische Rosen in Knospen. Erfurt: Nonne, 1738.

Wolf, Werner. »Aesthetic Illusion in lyric poetry?« Poetica 30.3/4 (1998): 251-289.

Zipfel, Frank. »Lyrik und Fiktion«. Handbuch Lyrik. Theorie, Analyse, Geschichte. Hg. Dieter Lamping. Stuttgart: Metzler, 2011. 162-166.

Zymner, Rüdiger. Manierismus. Zur poetischen Artistik bei Johann Fischart, Jean Paul und Arno Schmidt. Paderborn: Schöningh, 1995.

Zymner, Rüdiger. »Lyric and Its `Worlds« «. Journal for Literary Theory 11.1 (2017): 149-160.

Zymner, Rüdiger. Funktionen der Lyrik. Münster: Mentis, 2013.

Zymner, Rüdiger. Lyrik. Umriss und Begriff. Paderborn: Mentis, 2009. 